\title{
Laser beam propagation in non-linearly absorbing media
}

\author{
Andrew Forbes ${ }^{\#}$ \\ CSIR National Laser Centre, PO Box 395, Pretoria 0001, South Africa
}

\begin{abstract}
Many analytical techniques exist to explore the propagation of certain laser beams in free space, or in a linearly absorbing medium. When the medium is nonlinearly absorbing the propagation must be described by an iterative process using the well known Kirchoff-Fresnel diffraction equation. In this paper Gaussian and super-Gaussian beam shapes are propagated both through free space, and through a nonlinearly absorbing medium. The results show that shape invariance during propagation is dependent on the medium under consideration, and we find that super-Gaussian fields are shape invariant in certain nonlinearly absorbing media.
\end{abstract}

Keywords: Laser beam propagation, super-Gaussian beams, non-linear media

\section{INTRODUCTION}

Many processes in nature show non-linear dependences on the intensity of the laser beam, including such processes as Second Harmonic Generation, Third Harmonic Generation, Raman Scattering, Four Wave Mixing, and Multi-Photon Dissociation. The result is that the intensity distribution of the laser beams used in these processes is of the utmost importance. A Gaussian intensity distribution is desirable from a simple transportation point of view, whilst a flat top or super-Gaussian distribution will ensure a uniform interaction volume. It has been believed that the latter does not propagate in a mode that maintains its shape (intensity distribution function), and must be reshaped regularly to combat this problem. Thus beam delivery systems for applications in which long path lengths are needed (e.g., laser isotope separation) have traditionally been achieved with Gaussian beams using multiple pass cells of the Herriot design ${ }^{1,2,3}$. In this work it is shown that the absorption profile of the medium plays a very important role in determining the propagation characteristics of the laser beam in the medium. A short review of free space propagation is given in section 2 , and then the results for a purely theoretically constructed non-linear medium given in section 3 .

\section{FREE SPACE PROPAGATION WITH LINEAR ABSORPTION}

The propagation of Gaussian laser beams can be derived analytically, and is well understood. If the beam radius is defined as the distance at which the intensity is $1 / e^{2}$ of the peak intensity, then the change in radius during propagation in the $z$ direction may be expressed as

$$
w(z)=w_{0} \sqrt{1+\left(\frac{z}{z_{r}}\right)^{2}}
$$

where $w_{o}$ is the beam waist (minimum beam size) and set to be at $z=0$, and $z_{r}$ is the so called Rayleigh range - the distance at which the beam cross-sectional area doubles. The Rayleigh range may be expressed in terms of the wavelength of the light $(\lambda)$ as

$$
z_{r}=\frac{\pi w_{0}^{2}}{\lambda}
$$

\footnotetext{
\# aforbes1@,csir.co.za; phone +27 12 8412368; fax +27 128413152
} 
This, together with conservation of energy, gives rise to an analytical expression for the change in beam intensity profile during propagation:

$$
I(r, z)=\frac{2 P}{\pi w^{2}(z)} \exp \left(-2\left(\frac{r}{w(z)}\right)^{2}-\alpha z\right)
$$

Here we have assumed that the total power content of the laser beam is $P$, and that we have a circularly symmetric beam (with little effort the above may be written in terms of Cartesian co-ordinates). From these relations one can show that the divergence of the Gaussian beam is inversely proportional to the beam waist size, and from Equations (1) and (3) leads to a change in the width of the pulse during propagation, but not the intensity profile itself - i.e., the Gaussian beam remains Gaussian during propagation in free space. The second term in the exponent allows for linear absorption along the path length from $z=0$, and gives rise to a decrease in the peak intensity of the pulse, but again, not the size or spatial profile of the pulse.

More general laser beams - multimode beams for example, can also be described by Equation (1) so long as the beam size is defined as the second moment of the intensity, and the wavelength is increased by a factor $\mathrm{M}^{2}$ representing the so called beam quality factor: $\lambda \rightarrow \mathrm{M}^{2} \lambda$. This formalism is useful for understanding some aspects of how laser beams propagate, but is not useful in understanding how the laser beam intensity profile changes during propagation. In fact, the moments method of describing laser beams does not shed any light on the energy distribution of the pulse.

We will next consider the general case of flat-top beams. A flat-top beam is defined as having a constant intensity in some region, and zero everywhere else:

$$
I(r, z=0)=\left\{\begin{array}{cc}
I_{0}, & r \leq w_{0} \\
0, & r>w_{0}
\end{array}\right.
$$

A class of laser beam profile that can be used to link the Gaussian profile to that of the flat-top beam is the so called super-Gaussian profile

$$
I(r, z=0)=I_{0} \exp \left(-2\left(\frac{r}{w_{0}}\right)^{2 p}\right)
$$

where $p$ is some integer value. As the value of $p$ increases, so the function starts to approximate a flat-top beam. When $p=1$, the function is the standard Gaussian field. For this reason super-Gaussian functions are useful to analyze. They also have the advantage that the Flattened Gaussian Beam approximation ${ }^{4}$ can be used to generate useful data on the propagation characteristics of these beams. A linear absorption term may also be introduced to the FGB approximation by rewriting the wave vector as a complex number $k=k_{r}+k_{i}$, with $k_{i}$ representing either absorption $\left(k_{i}<0\right)$ or gain $\left(k_{i}>\right.$ 0 ), as was done in [5]. 


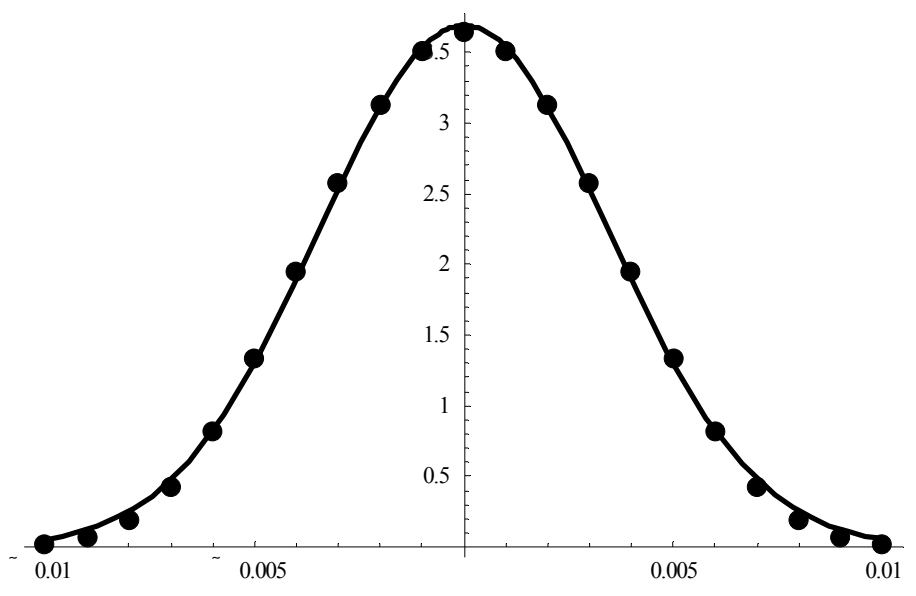

Figure 1: A super-Gaussian beam in the far field (data points) is very close to a Gaussian function (solid curve).

\section{PROPAGATION WITH NON-LINEAR ABSORPTION}

\subsection{Model}

The propagation of laser beams in a medium with non-linear absorption was tackled numerically in this work. The field was propagated through free space taking into account all diffraction effects:

$$
u_{1}(x, y, z)=\frac{1}{i \lambda} \iint_{S} u_{0}\left(x_{0}, y_{0}, z_{0}\right) \frac{\exp (i k R)}{R} \cos (n, R) d S
$$

Then the new field $u_{l}$ was modified according to the absorption profile under investigation. This was done by calculating the loss at each part of the field based on the absorption as a function of intensity. The new field was then again propagated through free space using Equation (6) and the process repeated. Thus the continuous absorption problem was solved by breaking the calculation into discrete absorption screens, placed near enough to one another that over extended distances the absorption would appear nearly continuous. Care was taken to make the step sizes between screens small enough to make the cascade almost continuous, but also the step size had to be made large enough to speed up the calculation. Typically a $1 \mathrm{~m}$ length would be split into 1000 such screens, while the laser beam radius was in the 3-5 $\mathrm{mm}$ range. This makes the distances between screens too small for any far field approximations to Equation (6) to be useful. "Calibration" tests were done to ensure the integrity of the code (written in ANSI C using National Instruments' LabWindows as the programming environment).

In order to compare two intensity profiles (say $I_{1}$ and $I_{2}$ ) to one another to see "how much they differ", a percentage root mean square test was performed

$$
\% r m s=100 \times \frac{\iint\left(I_{1}-I_{2}\right)^{2} d A}{\sqrt{\iint I_{1}^{2} d A \cdot \iint I_{2}^{2} d A}}
$$

This method allows a single number to be generated that gives an indication if the intensity profiles are similar or not. Such a measure means that the \%rms will increase if the profile and/or the size changes, and can result in a number greater than $100 \%$. A $0 \%$ value implies that both the size and the profile are identical. 


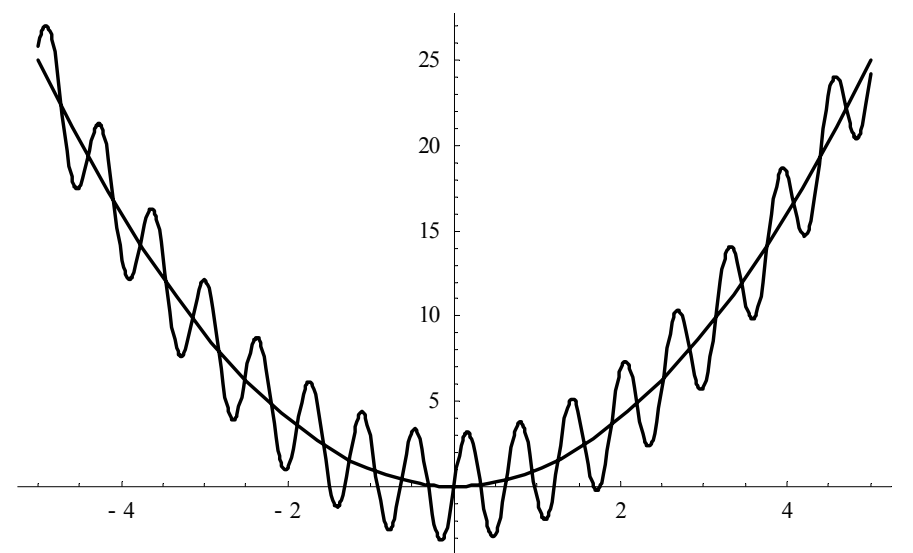

Figure 2: A \%rms value allows two arbitrary functions to be compared directly over a specified range.

\subsection{Results}

Figure 3 shows the intensity profiles of a $3 \mathrm{~mm}$ Gaussian and super-Gaussian beam propagated through the non-linearly absorbing medium. The intensity dependent absorption leads to a flattening of the Gaussian pulse during propagation something not observed in free space propagation with linear absorption. The super-Gaussian beam appears to maintain its shape and even its size during propagation, with only a decrease in the peak intensity evident due the loss of energy through absorption. Overlaid on the super-Gaussian data is the shape of the pulse after the same propagation distance if there were no absorption, indicating the dramatic change in propagation properties.
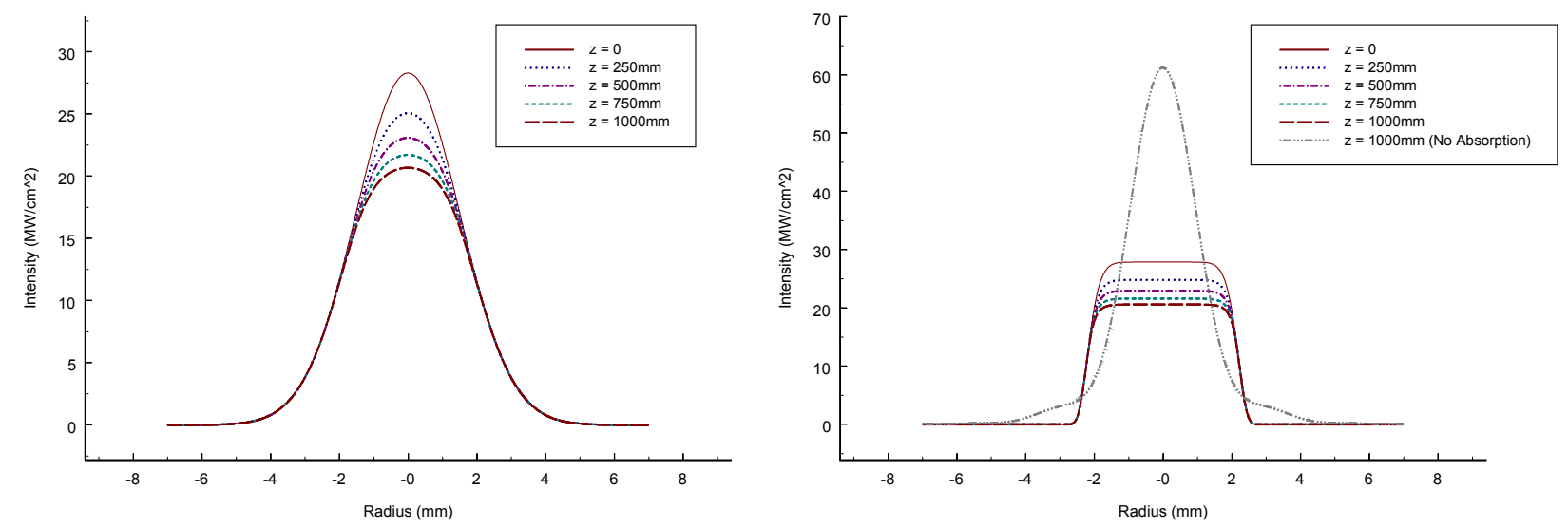

Figure 3: The change in intensity profile of a Gaussian (left) and super-Gaussian (right) beam after passing through a non-linearly absorbing medium. These results were first reported in [6], but not analysed in any detail.

One can offer an intuitive argument to explain this: as super-Gaussian beams propagate, so characteristic "spikes" and "ripples" appear on the profile. It seems that in the presence of non-linear absorption these intensity fluctuations are suppressed due to the fact that they are preferentially reduced through absorption.

Figures 4 and 5 show the \%rms change in the beam intensity function during propagation (with the intensity at $z=0$ taken as the reference), both for free space propagation and for propagation in a non-linear medium. The Gaussian beam (Figure 4) shows a much large change in the non-linear medium. The super-Gaussian beam shows the opposite. 
In these plots the intensity of each pulse was normalized to 1 in order to compare shape and size changes only, and not absorption changes.

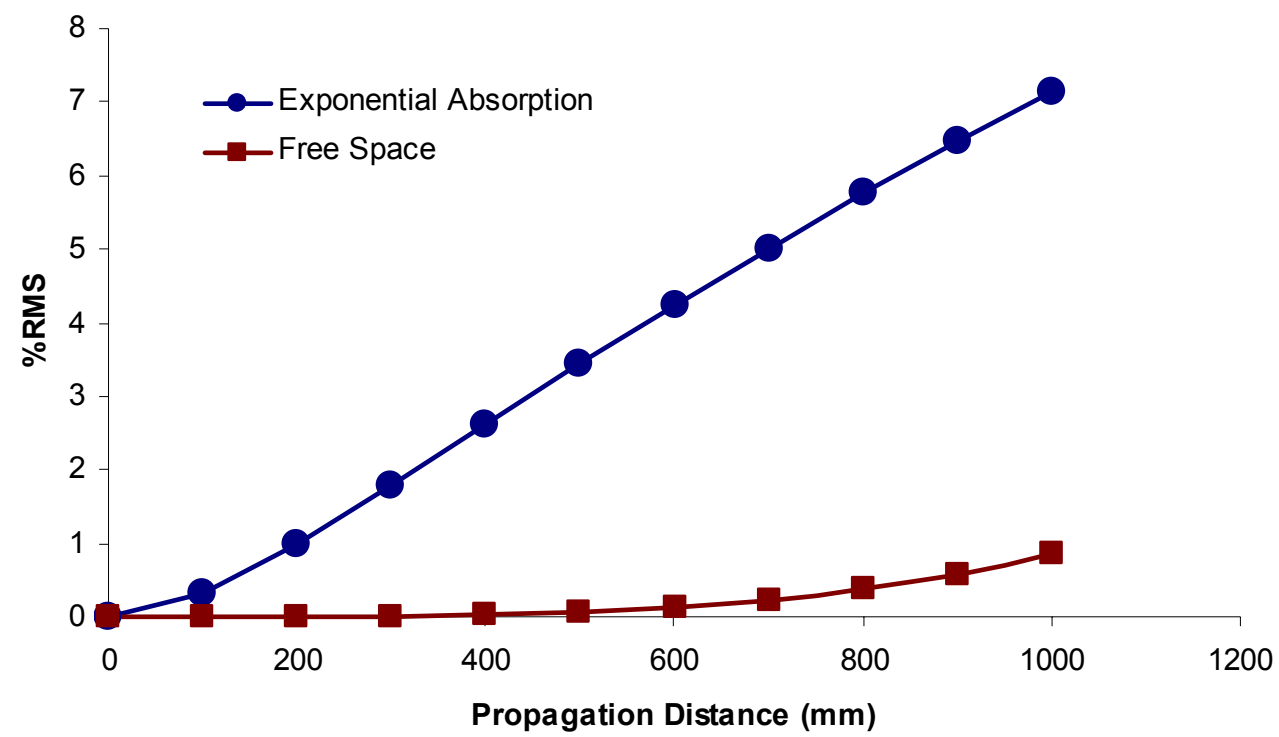

Figure 4: Data for a $w_{0}=3 \mathrm{~mm}$ Gaussian beam. In free space the profile does not change at all, and the \%rms change is only due to diffractive spreading. In a non-linearly absorbing medium, the Gaussian changes shape and flattens at the centre, resulting in an order of magnitude change in the \%rms value.

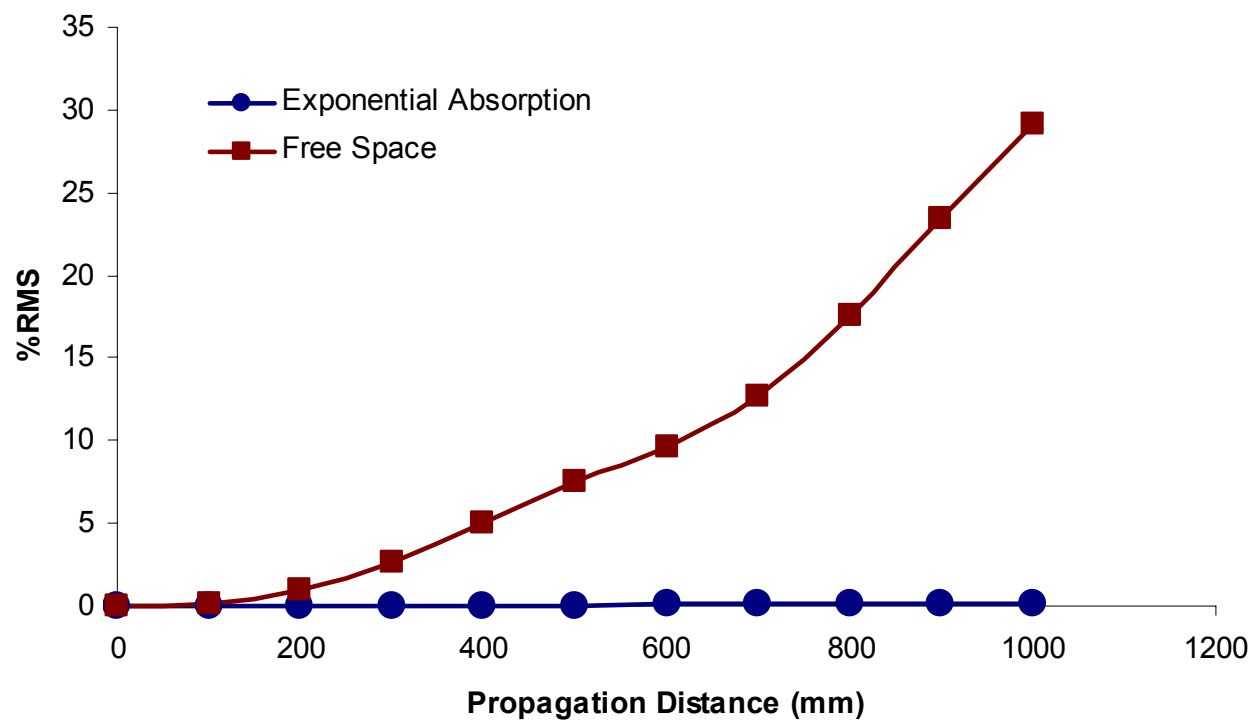

Figure 5: A $w_{0}=3 \mathrm{~mm}$ super-Gaussian beam showing a dramatically improved holding ability in the non-linear medium. This is a two orders of magnitude improvement in the \%rms value over the free space value for the same propagation distance. 


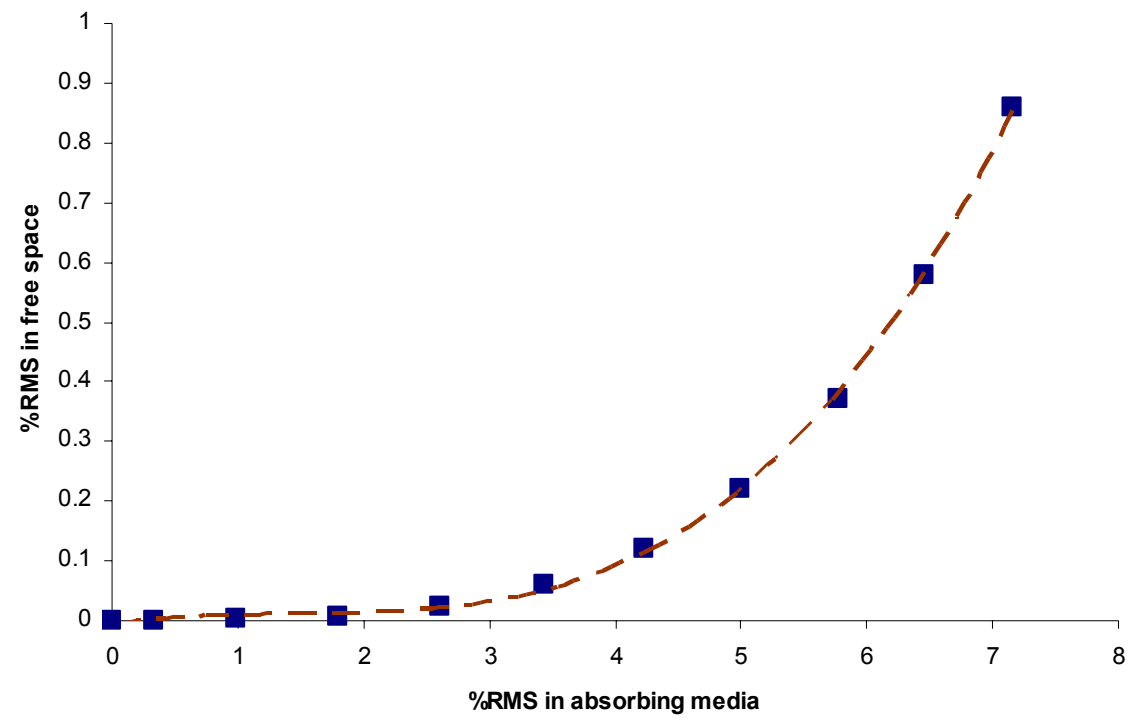

Figure 6: Parametric plot for a Gaussian beam of the \%rms change in free space propagation against that in an absorbing medium, for the same propagation distances.

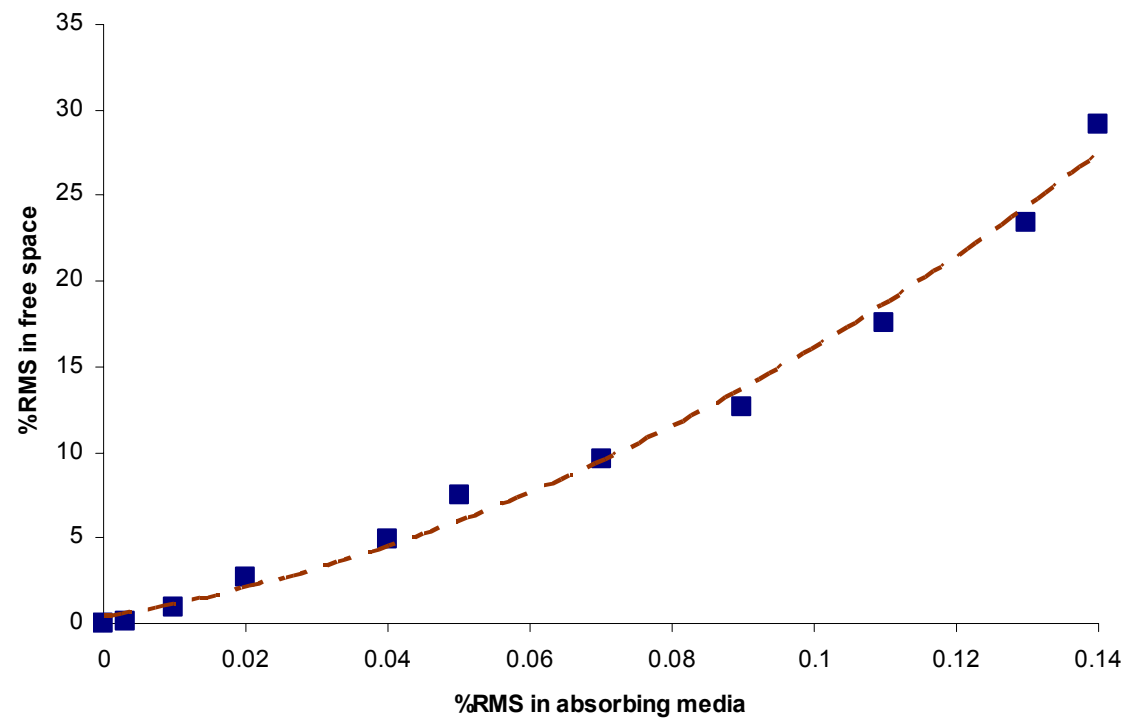

Figure 7: Parametric plot for a super-Gaussian beam of the \%rms change in free space propagation against that in an absorbing medium, for the same propagation distances.

In Figures 6 and 7 parametric plots are shown for both the Gaussian and super-Gaussian beams, with the free space beam changes plotted with the changes calculated in the absorbing medium. The scale on the two axes indicates that in the Gaussian case there is an order of magnitude increase in \%rms change when propagating in the non-linear medium as compared to propagating in free space. Conversely, the super-Gaussian beam shows roughly a two order of 
magnitude decrease in \%rms change when propagating in the non-linear medium as compared to propagating in free space.

\section{CONCLUSION}

Laser beams with Gaussian intensity profiles are understood to propagate shape invariant under free space conditions, or in mediums with linear absorption. Laser beams with Super-Gaussian intensity profiles however show rapid change in shape under free space propagation, and end close to Gaussian in the far field. However, when these beams are propagated in a medium with an absorption coefficient that is intensity dependent, then the super-Gaussian beam appears to remain shape invariant. This has implications in a number of fields, including that of isotope separation with laser beams. An intuitive argument has been presented to explain the results but at present experimental verification is lacking.

\section{REFERENCES}

1. W. Fub, J. Gothel, M.M. Ivanenko, K.L. Kompa, W.E. Schmid and K. White, "IR Multiphoton absorption and isotopically selective dissociation of $\mathrm{CHClF}_{2}$ in a Herriot multi-pass cell”, Z. Phys., D29, pp. 291-298, 1994.

2. H. Kojima, K. Uchida, and Y. Takagi, „, A waveguide reactor for IR multiphoton dissociation and its application to ${ }^{13}$ C laser isotope separation", Appl. Phys. B, 41, pp. 43-48, 1986.

3. J. Gothel, M.M. Ivanenko, P. Hering, W. Fub and K.L. Kompa, "Macroscopic enrichment of ${ }^{12} \mathrm{C}$ by a high-power mechanically Q-switched $\mathrm{CO}_{2}$ laser”, Appl. Phys. B, 62, pp. 329-332, 1996.

4. F. Gori, "Flattened Gaussian beams", Opt. Comm., 107, pp. 335-341, 1994.

5. B. Lu and S. Luo, "General propagation equation of flattened Gaussian beams", JOSA A, 17 (11), pp. 2001-2004, 2000 .

6. A. Forbes, H.J. Strydom, L.R. Botha and E. Ronander, "Beam delivery for stable isotope separation", Proc. SPIE 4770, pp. 13-27, 2002. 